\title{
A Granular System of Ellipses under Linear Shear
}

\author{
Dong Wang ${ }^{1, \star}$, Hu Zheng ${ }^{1,2, \star \star}$, and Robert P. Behringer ${ }^{1, \star \star \star}$ \\ ${ }^{1}$ Department of Physics \& Center for Nonlinear and Complex System, Duke University, Durham, NC, USA \\ ${ }^{2}$ School of Earth Science and Engineering, Hohai University, Nanjing 210098, China
}

\begin{abstract}
Shear of granular systems of disks (in $2 D$ ) and spheres ( $3 D$ ) has been studied extensively. However, less is known about systems of non-spherical particles, i.e., ellipses and polygons, etc. Here we studied a quasi $2 D$ granular system composed of ellipses in a novel apparatus, which provided homogeneous simple shear by utilizing the weak friction between ellipses and the deformable base of the apparatus. Shear jamming, which was first reported for a disk system [1], was also observed for our ellipse system. We contrast shear jamming for systems of disks and systems of ellipses. By tracking the positions and orientations of the ellipses, we observed that the ellipses gradually rotated to align along a preferred direction as the shear strain increased. We also applied Voronoï tessellation to the packing of ellipses, showing that the distribution of the local density changed during shear. By contrast, such a change in the distribution was not observed in a disk system [2].
\end{abstract}

\section{Introduction}

Granular systems have shown to be of great interest and benefit to theoretical study and industrial applications. Of special interest is the response of a granular system to shear. Systems composed of disks (in $2 D$ ) or spheres (in $3 D$ ) under shear have been studied extensively in theory and experiments, including simulations [1,3-10]. Though simple and able to capture important features of granular systems, disk/sphere models are often different from real situations, i.e., particles have an anisotropic or even irregular shape. Much is less known for this type of systems.

One of the most simple anisotropic shapes is an ellipse. There have been studies on ellipses, with comparisons to disks, i.e., jamming transitions and dynamical properties, both in experiments and simulations [11-14]. Most of these studies either focus on systems above jamming or considers dynamics. On the other hand, Bi et al. have shown that a granular system of frictional disks below the isotropic jamming point can be jammed by applying a certain amount of shear strain quasi-statically, a process called shear jamming [1]. Therefore, it is necessary to test shear jamming in a system of ellipses and compare the results with disks.

In this work, we use a system of quasi- $2 D$ elliptical particles made of photoelastic materials and apply quasistatic simple shear to it homogeneously. Note that simple shear can be decomposed in to a rotation followed by pure shear, which consists of compression in one direction and dilation in the perpendicular one. The photoelastic property of particles enable us to visualize and determine pres-

\footnotetext{
^e-mail: dw148@duke.edu

$\star \star$ e-mail: hz64@phy.duke.edu

$\star \star \star$ e-mail: bob@phy.duke.edu
}

sure on each individual particle. We find that shear jamming still occurs in a granular system of ellipses. Using UV bars on the surfaces of particles, we determine their centers and orientations. The evolution of the probability distribution of particle orientations shows that particles tend to align during shear to a direction that is perpendicular to the compression of shear. Furthermore, by applying Voronoï tessellation to the packing, we see a dramatic change and asymmetry in the distribution of the Voronoï cell areas, indicating a spacial inhomogeneity.

\section{Experimental Methods}

The granular system is composed of more than 3000 monodisperse ellipses made of photoelastic material, which reveal force information on individual particles if put between a pair of crossed polarizers. An example of a polarized image is shown in Fig. 1(c), with particles that bear forces lighting up and ones that are force free remaining dark. Each particle has dimensions of: major axis $1.04 \mathrm{~cm}$ and minor axis $0.57 \mathrm{~cm}$. We also obtain an image taken with unpolarized white light, as shown in Fig. 1(b). An invisible UV bar, only illuminated by UV light, was drawn at the center of the particle surface, as shown in Fig. 1(d), to accurately find the major axis orientation (indicated as orientation in the following text) and center of a particle.

To apply a homogeneous simple shear to the granular system, we used a bottom-assisted shear apparatus utilizing friction between the base and particles. The bottom is split into small slats of width $1.27 \mathrm{~cm}$, moving with the wall, to ensure that every particle feels the uniform shear profile. That is shear is applied homogeneously throughout the system, and not only from the boundaries. Such a 
(a)
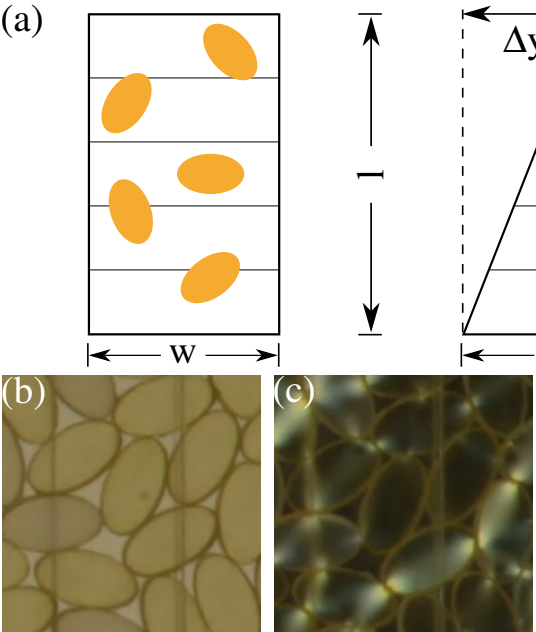
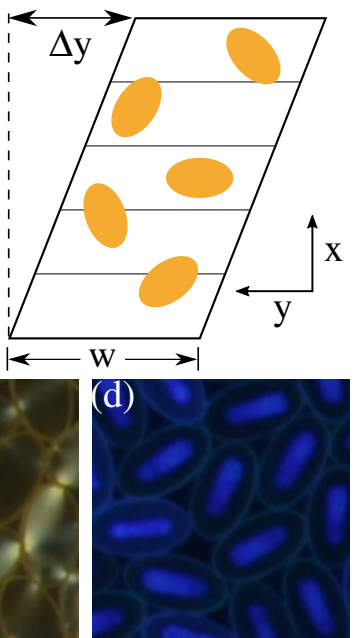

Figure 1. (a) Sketch of the shear cell setup with the Cartesian coordinate system illustrated. Starting as a rectangular box, which has a length $l=62.1 \mathrm{~cm}$ and a width $w=29.7 \mathrm{~cm}$, the cell is deformed to a parallelogram, with the deformation characterized by shear strain: $\gamma=\Delta y / l$. White light illuminates the shear cell from below while UV light illuminates the shear cell from above. Bottom: examples of images of the same part from a shear experiment taken (b) with white light on and without polarizer, (c) with white light on and with polarizer and (d) with white light off and UV light on.

design is sketched in Fig. 1(a). For a typical experiment, starting from a rectangular box of width $w=29.7 \mathrm{~cm}$ and length $l=62.1 \mathrm{~cm}$, the system was sheared along the width side by a small strain step, 0.0027 , followed by enough relaxation time for the particles to come to rest. Then we obtained three pictures in sequence: a polarized image to record forces, a normal and a UV image to determine positions and orientations of particles. Such a process of small amplitude shear, relaxation and image acquisition is repeated 100 times to achieve a total strain $(\gamma)$ of 0.27 .

\section{Results and Discussion}

\subsection{Shear Jamming}

Shear jamming is still observed in the case of ellipses, and that is qualitatively similar to what is observed for disks. According to the simulations [15], the isotropic jamming point, $\phi_{J}$, for a frictionless bidisperse ellipse system with an aspect ratio $1.82(=1.04 / 0.57)$ is between 0.885 and 0.89 . The densities we tested are all well below this value. Typical results from an example of $\phi=0.847$ (the same experiment for the results shown in Figs. 3 and 4) are shown in Fig. 2. Starting from a completely stress-free state, as shown in Fig. 2(a), where no particle is bearing any forces, the system gradually comes to a fragile state, where particles form a force network, force chains, mainly in the compression direction, as shown in Fig. 2(b) $[1,6]$. Such a special state is mechanically stable; however, it cannot sustain more than a small stress if the shear is reversal. This feature distinguishes itself from a jammed

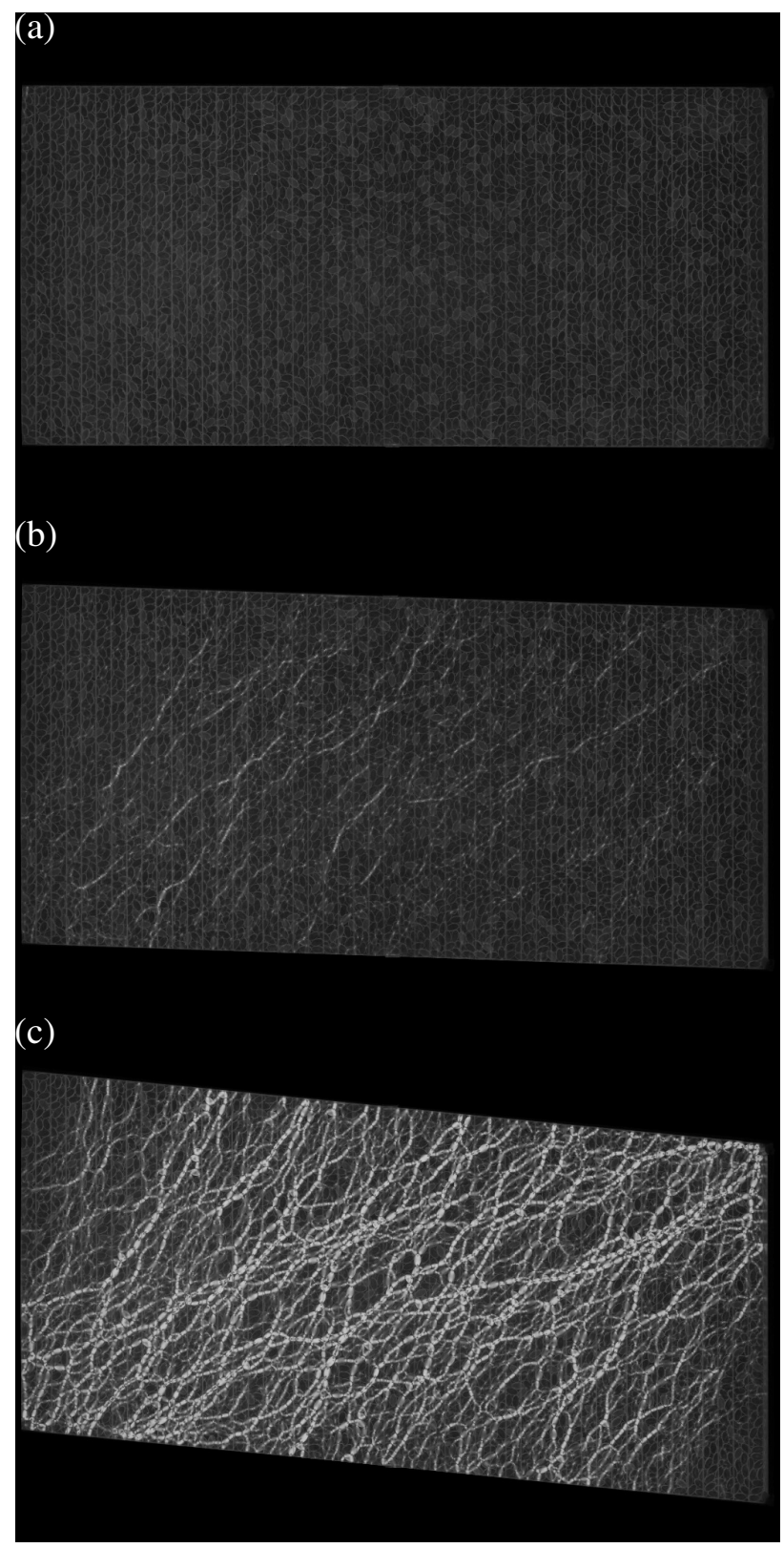

Figure 2. Three snapshots of polarized images from one shear experiment ( $\phi=0.847$ ) showing that the system undergoes transitions from (a) unjammed to (b) fragile and eventually to (c) shear jammed states. The corresponding strains from (a) to (c) are $\gamma=0,0.027,0.135$.

state, where the system can sustain stress from any direction. As $\gamma$ increases more, force chains in the compression direction bend and make extra contacts, and eventually percolate all over the system, bringing the system to a jammed state, as shown in Fig. 2(c).

In order to investigate the difference for shear jamming between disk and ellipse systems, we probe structural changes in the ellipse system and contrast it with disks. Specifically, we look at the probability distribution function (PDF) of orientations of ellipses, denoted as $\theta$ in the following, and apply Voronoï tessellation to the packing, with results shown and discussed below. 


\subsection{Orientation Distribution}

When computing the probability distribution function of the orientations of ellipses, only the ellipses whose distances to the nearest boundary are twice or more the minor axis are taken into consideration to minimize the boundary effect. After such an elimination of boundary particles, approximately $2500 \sim 3000$ particles are used in the calculation, which still gives reliable statistical results.

For the ease of plotting, $\theta$ is confined in the range $\left(0^{\circ}, 180^{\circ}\right)$ and then extended to $\left(0^{\circ}, 360^{\circ}\right)$ by adding $180^{\circ}$ to the original values, where $\theta=0^{\circ}$ corresponds to the positive $x$ axis in the inset of Fig. 1(a). Results from a few selected $\gamma$ are shown in Fig. 3. Note at $\gamma=0$ in Fig. $3(\mathrm{a})$, there are more ellipses oriented around $80^{\circ}$, while supposedly there will be no preferred orientations if particles are put randomly. The main reason is: in order to achieve a stress free state at a relatively high $\phi$, particles tend to align with their neighbors, which hence creates a local order. However, such a built-in order does not affect the final conclusion, as seen in the following.

As $\gamma$ increases, we can clearly see that: first, the distribution remains non-uniform; second, the most probable $\theta$ changes from $\sim 80^{\circ}$ at $\gamma=0$ to $\sim 110^{\circ}$ with a slightly higher probability at $\gamma=0.15$, and then stays around the same value for the rest of shear. The first feature indicates that shear induces or keeps a preferred direction in an ellipse system. The second feature indicates that the system reaches a steady state. Such a feature is reminiscent of the work by Börzsönyi et al., where they showed a granular system of elongated particles formed a certain angle with the streamline of shear, unaffected by the shear rate [14]. Furthermore, both features show up for all the other densities tested.

As mentioned in Sec. 1, there exists a rotation associated with simple shear. The amount of rotation is given by $\tan ^{-1}(\gamma / 2)$, which in our case is no larger than $\tan ^{-1}(0.27 / 2)=7.7^{\circ}$. From Fig. 3, we clearly see that the change of the preferred $\theta$ is more than $15^{\circ}$. Therefore, such a change cannot be only induced by the rotation associated with shear. Note that an ellipse is more stable if the forces it bears are mainly perpendicular to its major axis. Combining this with the fact that shear induces force anisotropoy with larger forces mainly in the compression direction, particles are naturally expected to align their major axis perpendicular to the compression direction. This expectation coincides with our observation that the most probable $\theta$ is around $110^{\circ}$, which is very close to the perpendicular direction of compression direction $\left(45-\tan ^{-1}(\gamma / 2)+90^{\circ}=135^{\circ}-\tan ^{-1}(\gamma)\right.$, which is $120^{\circ}$ for $\left.\gamma_{\max }=0.27\right)$.

\subsection{Voronoï Tessellation}

To further verify the structural change of the system under shear, a Voronoï tessellation is applied to the packing. In this approach, we use the distance transformation tool in Matlab to determine a Voronoï tessellation associated with a particle, in which the distance of any point to that particle is less than that to any other particle [16]. We follow the

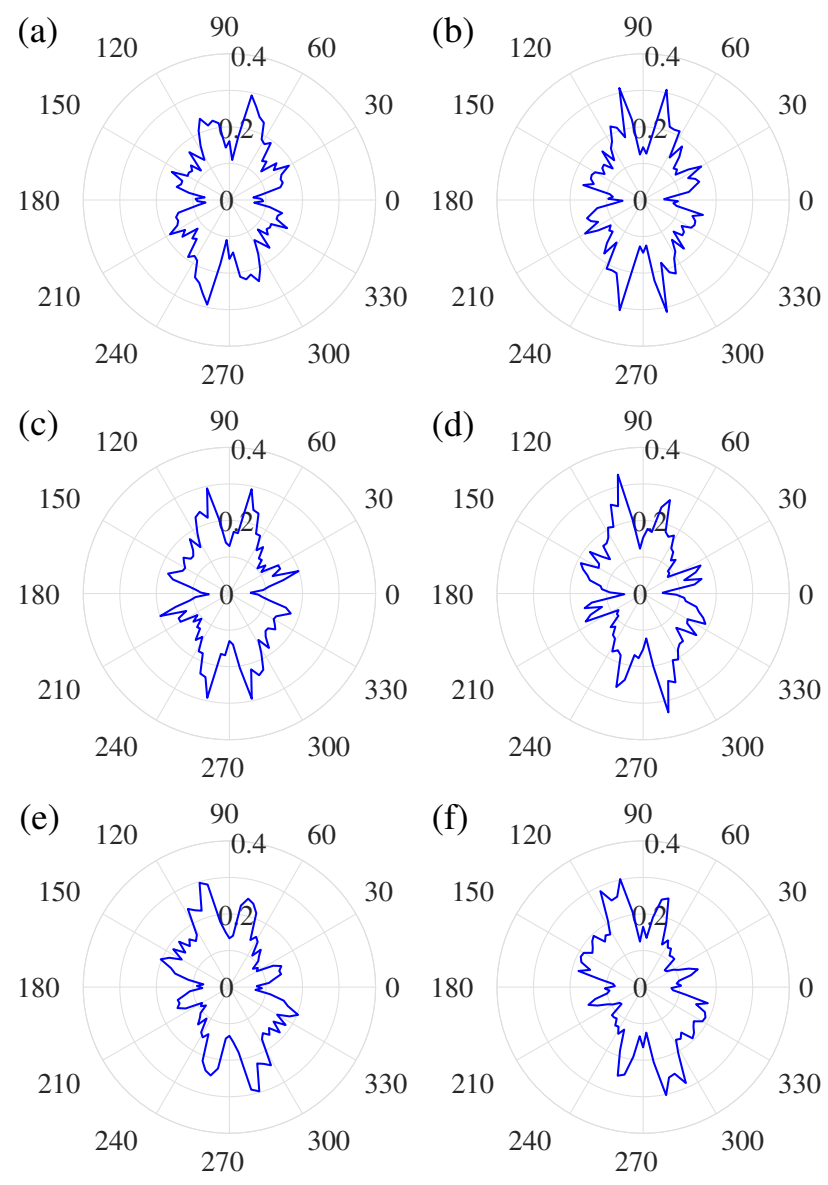

Figure 3. The probability distribution function of the orientation of ellipses from the same experiment in Fig. 2, excluding particles that are close to the boundary at different strain $\gamma$ : (a) $\gamma=0$, (b) $\gamma=0.0513$, (c) $\gamma=0.1026$, (d) $\gamma=0.1539$, (e) $\gamma=0.2052$, and (f) $\gamma=0.2565$. In the figures, the orientations are first fixed to the range $\left(0^{\circ}, 180^{\circ}\right)$ and then duplicated by adding $180^{\circ}$ to have a range in $\left(0^{\circ}, 360^{\circ}\right)$.

same procedure in Sec. 3.2 to minimize boundary effects, and the results are shown in Fig. 4.

Fig. 4(a) shows the evolution of the PDF of the Voronoï area, Avoro, with shear. The peak value, $P_{\text {max }}$, and corresponding Avoro, of the PDF, shift to a lower value as $\gamma$ increases. In comparison, Ren et al. reported that this PDF did not change during shear for a disk system [2]. In addition, Aste et al. showed an invariant Gamma distribution of the Voronoï volume in static granular spheres of different packing fraction by rescaling the Voronoï volume [10]. Here we applied the same rescaling: $A_{\text {rescaled }}=\left(\right.$ Avoro $\left.-A_{\min }\right) /\left(\langle\right.$ Avoro $\left.\rangle-A_{\min }\right)$, where $A_{\text {min }}$ is the minimum and chosen to be 1.048 times the actual area of an ellipse. Assuming the data to be the Gamma distribution, given by

$$
P\left(A_{\text {rescaled }}\right)=\frac{1}{\Gamma(k) \theta^{k}} A_{\text {rescaled }}^{k-1} e^{-A_{\text {rescaled }} / \theta},
$$

$k$ and $\theta$ can be determined by the mean $(k \theta)$ and variance $\left(k \theta^{2}\right)$. One thing to note is that due to the rescaling, the mean is always 1 , namely, $k \theta=1$. 

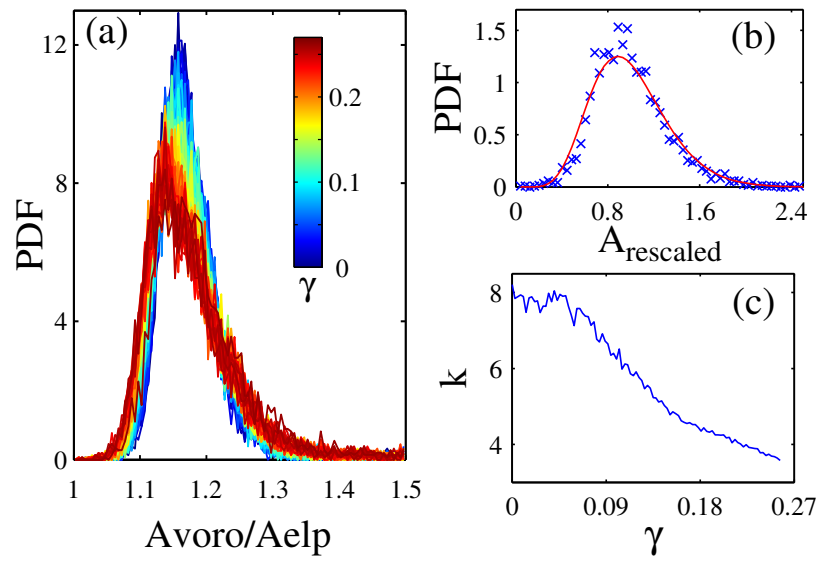

Figure 4. (a) Probability distribution function (PDF) of the Voronoï area of ellipses in the unit of the area of an ellipse, Avoro/Aelp, from the same experiment in Fig. 2, as a function of shear strain $\gamma$, indicated by different colors. The inset shows the color bar for $\gamma$. (b) The Gamma distribution fit (red line) to the original data (blue crosses) at $\gamma=0$ for the rescaled Voronoï area: $A_{\text {rescaled }}=\left(A-A_{\min }\right) /\left(\langle A\rangle-A_{\min }\right)$. (c) The evolution of $k$ from the Gamma distribution fit as $\gamma$ increases.

An example of the Gamma distribution fit is shown in Fig. 4(b) for $\gamma=0$. Since $k \theta$ is fixed regardless of $\gamma$, we only look at $k$ here, plotted against $\gamma$ as shown in Fig. 4(c). Starting from a value around $8, k$ stays the same for small $\gamma$ and then gradually decreases as $\gamma$ increases. The skewness of the Gamma distribution is proportional to $1 / \sqrt{k}$, hence the PDF of Avoro becomes more and more asymmetric as $\gamma$ increases.

\section{Conclusion and Outlook}

To summarize, applying homogeneous shear to a quasi $2 D$ granular system of ellipses causes shear jamming for an ellipse system well below isotropic jamming point. Under applied strain, particles tend to align with their major axes approximately perpendicular to the compression direction of shear. Such an alignment is seen in the probability distribution of the orientations. A structural inhomogeneity, indicated by this alignment, is further validated by the probability distribution of the Voronoï cell area, where the most probable value shifts to a smaller value. By contrast, such a inhomogeneity induced by shear is not observed with disk granular systems. Therefore, by introducing anisotropy into the shape of elements that make up the packing, the response of the packing shows a substantially different behavior, especially structurally.

We note that Schaller et al. showed an application of Voronoï tessellation in frictional ellipsoids packing to explain the local origin for global contact number [12], which can serve as a criterion to distinguish jammed states from unjammed ones. For the future work, we would like to apply similar analysis to see if there exists a connection between Voronoï area and contact number, and hence shear jamming. In addition, a more detailed analysis of force networks is required to better distinguish ellipses from disks during the shear jamming transition.

\section{Acknowledgments}

This work was supported by NSF grant DMR1206351, NASA grant NNX15AD38G and the William M. Keck Foundation. DW and RB would like to thank a Triangle MRSEC fellowship. HZ thanks NSFC Grant No. 41672256 and the NSF (Jiangsu, China) Grant No. BK20140845 for financial support.

\section{References}

[1] D. Bi, J. Zhang, B. Chakraborty, R.P. Behringer, Nature 480, 355 (2011)

[2] J. Ren, J.A. Dijksman, R.P. Behringer, AIP Conference Proceedings: Powders \& Grains 1542, 527 (2013)

[3] S.F. Edwards, R.B.S. Oakeshott, Physica A 157, 1080 (1989)

[4] A.J. Liu, S.R. Nagel, Nature 396, 21 (1998)

[5] C.S. O’Hern, L.E. Silbert, A.J. Liu, S.R. Nagel, Phys. Rev. E 68, 011306 (2003)

[6] M.E. Cates, J.P. Wittmer, J.P. Bouchaud, P. Claudin, Phys. Rev. Lett. 81, 1841 (1998)

[7] T.S. Majmudar, R.P. Behringer, Nature 435, 1079 (2005)

[8] T.S. Majmudar, M. Sperl, S. Luding, R.P. Behringer, Phys. Rev. Lett. 98, 058001 (2007)

[9] L.E. Silbert, Soft Matter 6, 2918 (2010)

[10] T. Aste, T. Di Matteo, M. Saadatfar, T.J. Senden, M. Schröter, H.L. Swinney, EPL 79, 24003 (2007)

[11] M. Mailman, C.F. Schreck, C.S. O'Hern, B. Chakraborty, Phys. Rev. Lett. 102, 255501 (2009)

[12] F.M. Schaller, M. Neudecker, M. Saadatfar, G.W. Delaney, G.E. Schröder-Turk, M. Schröter, Phys. Rev. Lett. 114, 158001 (2015)

[13] J. Tang, R.P. Behringer, EPL 114, 34002 (2016)

[14] T. Börzsönyi, B. Szábo, G. Törös, S. Wegner, J. Török, E. Somfai, T. Bien, R. Stannarius, Phys. Rev. Lett. 108, 228302 (2012)

[15] C.F. Schreck, N. Xu, C. O’Hern, Soft Matter 6, 061305 (2012)

[16] N.N. Medvedev, Dokl. Akad. Nauk 337, 767 (1994) 